\title{
Looking deeper into zeolites
}

\author{
Avelino Corma, professor at the Institute of Chemical Technology (ITQ-CSIC-Polytechnical University of Valencia), \\ talks to Nature Materials about challenges facing zeolites, and issues faced in commercializing research.
}

\begin{abstract}
Please tell us about your scientific background and why you research zeolites? My scientific background is on heterogeneous catalysis. My objective has always been to understand the reaction mechanism and catalytic active sites, to progress catalyst synthesis, and to maximize well-defined single or multiple isolated sites. Starting from those premises, zeolites are a natural selection. They are crystalline materials with well-defined pores and cavities. By being crystalline, we can introduce well-defined single or multiple active sites in framework or extra framework positions. Furthermore, we can use a variety of physicochemical characterization and modelling techniques to visualize those sites and their interaction with the reactants, and to establish structure-reactivity correlations. Finally, since zeolites have pores that match the dimensions of reactants, we can take advantage of confinement effects and their impact on reactivity.
\end{abstract}

During my PhD, I was working with amorphous silica-alumina catalyst and it was at the end of the $\mathrm{PhD}$ when I started to look into zeolites. They are crystalline silicoaluminates, with all the advantages named above. At that time, besides some work in academia - such as Professor Barrer in the UK - the biggest research effort on zeolites was in industry, and it was their application for fluidized catalytic cracking that made this field explode. Several companies subsequently established research groups of extraordinary high quality. They started to produce new zeolites, which led to many new scientific questions arising and the opening of a new research field with substantial industrial impact that continues to be very relevant today.

\section{Has the recent COVID-19 pandemic} affected your research?

It has certainly affected our research. Though it has slowed down our research in the lab, it has given me some peace. I have more time to think and meditate, which means that some of the everyday pressure has been released. This favours the generation of new ideas. On the other hand, it was a bit frustrating not to be able to check right away these ideas.

For our PhD students it was very annoying to delay their work. However, they

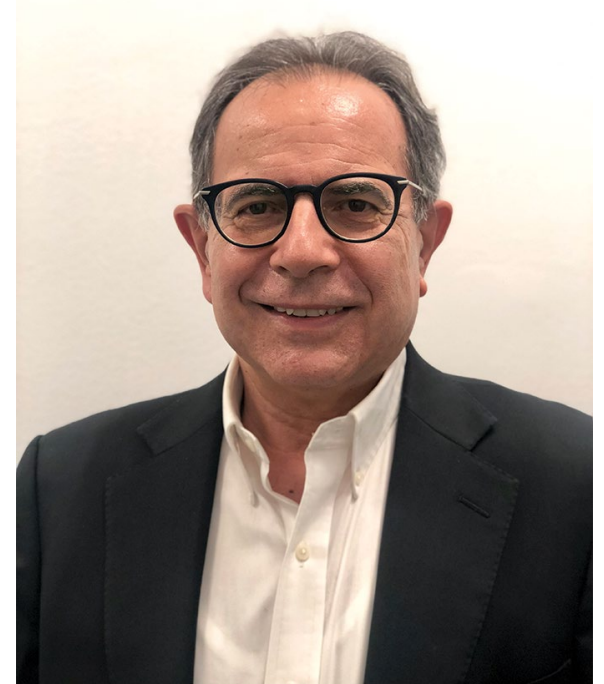

reported. The question now is how to synthesize a zeolite that does not exist. Here, we come back to the fundamental understanding of nucleation and crystallization.

For catalysis with zeolites, how we operate today is as follows. Imagine that you want to catalyse a reaction by using a zeolite. Then, we make use of accumulated knowledge and analogies, go into the toolbox of synthesized zeolite structures, and select several that look promising. Then, they will be tested to find the best candidate, and try to find an explanation on why that particular zeolite is the best. It appears to me that it would be more intellectually rewarding if one can perform the $a b$ initio synthesis of a zeolite for a pre-established reaction, while locating the active site in the appropriate position within the framework.

To do that, one can look first into the have had the opportunity to read and to generate their own ideas, which will inform their future research.

\section{What do you think are the current challenges for the field?}

Important challenges are to understand the fundamental mechanisms of how nucleation and crystallization occur in zeolites, and how we can control them. Up to now, most of the advances have been achieved by the slow accumulation of knowledge, chemical intuition, and trial and error.

Another challenge is how to locate active sites in specific pre-established positions within the zeolite. Imagine, for instance, that you have a zeolite with pores of different sizes. From the reactivity point of view, you may want to locate the catalytically active sites within one channel and not the other. You may even want to have one type of site in one channel and another site in the other channel to carry out tandem reactions. Going further, it is also challenging to control the sizes and chemical compositions of active species within the microporous zeolite environment. Locating active sites in preselected positions is certainly a fundamental and intellectual challenge, with important industrial implications.

A third challenge is to synthesize a predefined structure. Many thermodynamically feasible zeolites have been reaction mechanism and find a potential transition state (TS). Since the desired zeolite catalyst should minimize the energy of the TS by optimizing its interactions with the framework, one can now prepare a mimic of the reaction TS and use it as a template or structure-directing agent (SDA) for zeolite synthesis. If a zeolite is synthesized with this mimic SDA, it does not matter if it is a new zeolite or an already existing one, it should maximize interactions between the framework and TS mimic. This will result in a decrease of the activation energy with an increase in reaction rate. Therefore, the zeolite obtained with the TS mimic acting as a zeolite template should be, in principle, adequate for catalysing the desired reaction. This is like if one would be doing an imprinting of the TS on a solid and the result would be analogous to producing solid catalytic antibodies.

Finally, achieving catalytic enantioselectivity with zeolites is also a challenge. In my opinion, even better than achieving chiral zeolite structures, the objective would be to produce chiral centres within the zeolite framework.

As you can see, we have very interesting challenges and to achieve these objectives will require teams able to develop and put together advanced synthesis, characterization, modelling and catalytic reactivity techniques. 
You have successfully filed many patents. Is there a tension between publishing scientific research and submitting patent applications?

The main tension is that we cannot publish the results until the patent has been revised and, at least, filed. This increases the time of publication. Then, when the results are very new and with important implications, we start to get nervous. This is even more so when working with a company that wishes to further protect the discovery with additional patents.

I had some bad moments when the company wants to keep this knowledge and did not allow us to publish the work. Fortunately, this is not common.

\section{What are the differences in writing} for each format, and indeed what are the advantages and disadvantages in pursuing 'commercial' research versus 'purely intellectual' research?

You have to be much more detailed in the scientific paper, with an explanation of, the results obtained on the basis of an initial hypothesis. In the case of the patent, what matters is the result, no. explanation is required. In a patent one has to be careful what you write or how you write it to avoid conflicts with other patents or publications that can invalidate your claims.

Usually the companies we work with have very good patent lawyers. We give them a draft of the patent or a detailed report on the work. After the exchange back and forth of a few versions, they end up producing a very good and protective document.

With respect to industrial versus 'intellectual' research, I would not establish differences based on those terms. In our case, we first set scientific challenges. If we are successful, we try to go further and see how our findings, can be used to solve an industrial problem. Then we make a proof of principle and, if successful, we either apply for a patent or we approach a company and try to develop it further with them. In that sense, our collaboration with industry has been, for most of the time, rewarding. Companies have very good research teams from which we also learn when working together.

In many publications, the authors claim in the introduction that even if their work is of more fundamental interest, if successful, it can help solve an industrial problem. The philosophy established in our institute since its foundation was to cover from the 'intellectual' part to the proof of principle. Perhaps that is the reason why there are about ten processes in which the catalysts were conceived in our institute. Not too many can say that they publish the concept, got the patent, and that industrial plants use that research.

Are there any specific issues with the practical technological application of zeolites that are not faced by other materials? Are these issues that could be aided by theoretical or computational insight? The scientific methodology used in zeolites research is not too different from that used for other catalysts. We have to synthesize the structure that maximizes the active sites required for the desired reaction, while avoiding the generation of sites that catalyse unwanted reactions. This is common for any catalyst. For zeolites, we have the added value of the porous structure with the corresponding shape selectivity and confinement effects. A tremendous advantage of zeolites is that, by being crystalline, you can model not only the structure but also the different elements that can be introduced in the framework or extra framework positions. Then, when combining modelling with operando spectroscopy and microkinetics we can study how the reaction occurs on the active sites and how we can also influence the reactivity by diffusion, adsorption and confinement. However, when approaching a catalyst manufacturer there are questions to be answered on how easy it is to implement zeolite synthesis with the tools they have, and how do the economics look taking into account technical benefits with respect to competing technologies and the manufacturing cost of these new zeolites.

This very cold exercise can be frustrating for a scientist that has a new zeolite that can do better than others used commercially, but cannot pay for the additional expenses in manufacturing. However, from time to time we can be close enough to pursue the objective. Moreover, what is not seen today as an opportunity can be seen tomorrow as a key technology for a different paradigm. This is the beauty of research: it increases human knowledge and so helps to solve present or future problems.

In our experience there is a continuous dialogue when working in with a company. You may come up with a new idea, but the material is too expensive, maybe because of the SDA used for synthesis. Then, you have to make an hypothesis on the synthesis in order to make it cheaper, and here comes again the research.

Zeolites are famous for having millions of potential structures but only 232 frameworks can be synthesized. Is this a limiting factor, or are there a vast amount of possibilities in the toolbox? When I was talking about the zeolite toolbox, I referred to structures that have been already synthesized. There are many more thermodynamically feasible zeolites. Unfortunately, we still do not have the fundamental knowledge to synthesize any pre-established specific structure.

Do you think zeolitic catalysis of petrochemicals will have a long-term future? Are there breakthroughs in other areas (such as biomass reforming) that are coming, and will they have a great impact?

Zeolites are already very successful beyond making fuels from oils, they also make a large number of other chemicals. Certainly, we use oil and natural gas for making organic chemicals. Another source of carbon is biomass, and zeolites are playing their unique role in this field. We are also seeing zeolites in process for making chemicals from synthesis gas and even from $\mathrm{CO}_{2}$ and $\mathrm{H}_{2}$ through constructing bifunctional catalysts. Moreover, zeolites are very successful catalysts for the elimination of contaminants such as $\mathrm{NO}_{x}$. They are extremely stable materials, and if their pore size can be enlarged without compromising stability, their possibilities for adsorption and catalysis will be greatly expanded.

I believe zeolites will continue to play an important role for sustainable catalytic processes. Chemical selectivity is also a key word in the circular economy and zeolites can be constructed to achieve unprecedented selectivities in catalysis and separations.

What can young scientists look to gain from specializing in research on zeolites? I would say that zeolites are ideal catalysts, from the point of view of learning. They bring together synthesis, characterization, reactivity, absorption and separation skills. It is an excellent field for enabling and young scientists to develop a strong foundation, since they will have to deal with physical chemistry, inorganic chemistry, organic chemistry and chemical engineering. Then, they can go into academia, but they will also be highly appreciated in industry.

Some of the $\mathrm{PhDs}$ and postdocs who have worked with me are now professors in universities or are working successfully in companies. A few are entrepreneurs, who started their own companies.

I always thought my role was to bring the students to the limit of their possibilities. It is not easy and it looks like I am sometimes a very tough guy, but later they appreciate what I am trying to do.

\section{Interviewed by Stephen Shevlin}

Published online: 21 September 2020 https://doi.org/10.1038/s41563-020-0787-4 